\title{
Mathematical model of interaction of flat copier with the residues of haulm of sugar beet
}

V. Adamchuk,

Dr. Sc., academician of NAAS,

National Science Center "Institute of Mechanization and Electrification Agriculture"

V. Bulhakov,

Dr. Sc., academician of NAAS,

I. Holovach,

Dr. Sc., Prof,

National University of Bioresources and nature use of Ukraine

Ye. Ihnatiev

Tavria state agrotechnological university

The purpose. To determine optimum design and kinematic parameters of copier cutter of heads of root crops on root on the basis of building mathematical model of interaction of flat passive copier with heads of root crops. Methods. Methods of construction of mathematical models of operation of farm machines and their end-effectors with the use of original positions of mathematics, theoretical mechanics, development of programs and numerical calculations on the PC. Results. Findings of investigation in the process of interaction of passive copier with a head of root crop of sugar beet, and also results of numerical modeling of the developed systems of differential equations for determination of power and design parameters with the purpose of optimization of kinematic and design parameters of copier are resulted. Conclusions. The introduced power scheme of interaction of passive copier and orbicular surface of a head of root crop has created premises for development of the theory of process of interaction of passive copier with a head of root crop of sugar beet in view of elastic-damping properties of the residues of haulm.

Key words: sugar beet, harvesting, haulm, copier, equation of movement, mathematical model, design parameters.

\section{Formulation of the problem}

Advanced technology for harvesting sugar beet before the root crop is dug from the ground, the initial execution of the cut of the main mass of the hook and the subsequent final cutting of the heads at the root. Therefore, the effective functioning of most beet-harvesting machines depends on how precisely copying of the root crops will be made. This applies to most hoeing machines, roothead cleaners, trimmers, excavating working structures of some structures. In the world of practice a wide variety of mechanical devices have been widely used to copy beet root roots at the root and install cutting or cleaning working bodies at the required working height. These include passive copiers, active (drive, drum), and combined. However, the requirements of the required level of quality of work in different conditions of collection, simplicity of construction, metal intensity and energy intensity are not always fulfilled.

Analysis of last publications

Many published scientific papers [1-6] are devoted to the removal of the remains of a sugar beet stick after its continuous cutting at the root of the cutting apparatus. The results of investigations of roothead head cleaners of various designs are presented in them: lobed [1, 4, 6], ring [5], sector [7] and drum [2] types, as well as paraboloid [3]. Technological processes of collecting huckster of sugar and fodder beet 
are in many respects similar, but only experimental work [5, 9] is devoted to the research on harvesting fodder beet hinges.

Theoretical studies of the motion of the passive comb copier on the head of sugar beet root crops have also been thoroughly considered [10], but the results are mainly based on the use of geometric dependencies, and the mathematical model of this process is not developed.

In the paper [1], the shock interaction of a plane passive copier, inclined in a longitudinal-vertical plane with a head of sugar beet root, located immovably in the ground, is considered. The presented theory considers a special case of such interaction, when the head of the root crop is devoid of the tops remnants and is considered to be absolutely solid.

It should be emphasized that at this time in the world the most common trenchers of sugar beet root crops were widespread from the remnants of the hinges, which consist of mounted on one frame on the front of the comb copier and a flat knife behind it. However, as the results of comprehensive multi-year tests of such constructions of the use of the trimmers, in this case, comb copiers, show that the short remains of the hinges (petioles) tend to fall into the intervals between the comb copes and it directly contacts the head of the root. This leads to the fact that the comb causes deep traces to the bodies of sugar beet root crops and establish a higher cutting height. This leads to the fact that, when cutting the heads of sugar beet root crops, up to 6 ... $8 \%$ sugar content is lost, vertical cracks are formed in the upper parts of the root crop's bodies, due to the increased cut area, loss of sugorous raw materials is more intense [14, 15]. The use of simple conveyors leads to a speed of more than $2.0 \mathrm{~m} \cdot \mathrm{s}^{-1}$ before the periodic jerking, which leads to poor-quality circumcision of some root crop heads.

The above disadvantages can be eliminated by the use of new-design conveyors when flat-copy copiers are used in their schemes.

The aim of the study

Determination of optimal constructive and kinematic parameters of the copier device of the headers of root crops on the basis of the creation of a mathematical model of the interaction of a plane passive copier with heads of roots with their pruning at the root.

Research methods

Methods of constructing mathematical models of functioning of agricultural machines and their working bodies on the use of the basic provisions of mathematics, theoretical mechanics, programming and numerical calculations on the PC are used..

Results of research

Proceeding from the statistical dimensional-mass characteristics of sugar beet root crops and their location in the rows of crops, some of the basic design parameters of a trimming can be taken constructively or calculated according to known rather simple dependencies.

In conditions that are closest to real, the interaction of a plane passive copier at an initial instant occurs in fact with short and elastic bundles of hinges that remain on the lateral and central surfaces of the spherical head of the root after a continuous cut of the hinges by the rotating working body. Therefore, this interaction will no longer be rigid if you take into account the elastic and damping properties of the remains of the hinge.

Let's make an equivalent scheme of interaction of a plane passive copier, which is set at an angle $\alpha$ of inclination to the horizon, moves progressively $v_{p}{ }^{-}$at a rate and, when it comes to the head of the sugar beet root, initially begins to contact the elastic remnants of the hinges, which are located on the lateral spherical surface of the head of the root (Fig. 1).

In this case, the root sugar of sugar beet is modeled in the form of a rigidly fixed conic in the soil, and the upper part of it - the head protrudes to a certain height above the level of the surface of the soil and is conventionally considered in a longitudinal and vertical plane by a circle of radius $R$. The center of the head of the root is designated by a point $O$. 
The elastic and damping properties of the hinged bales on the head of the root of the beet on the equivalent scheme are shown in the form of an elastic-damping model with corresponding coefficients $c$ stiffness and $\mu$ damping. The interaction of a flat copy with the indicated root crop model and the remnants of a gimbal on its head is carried out at the point $A$.

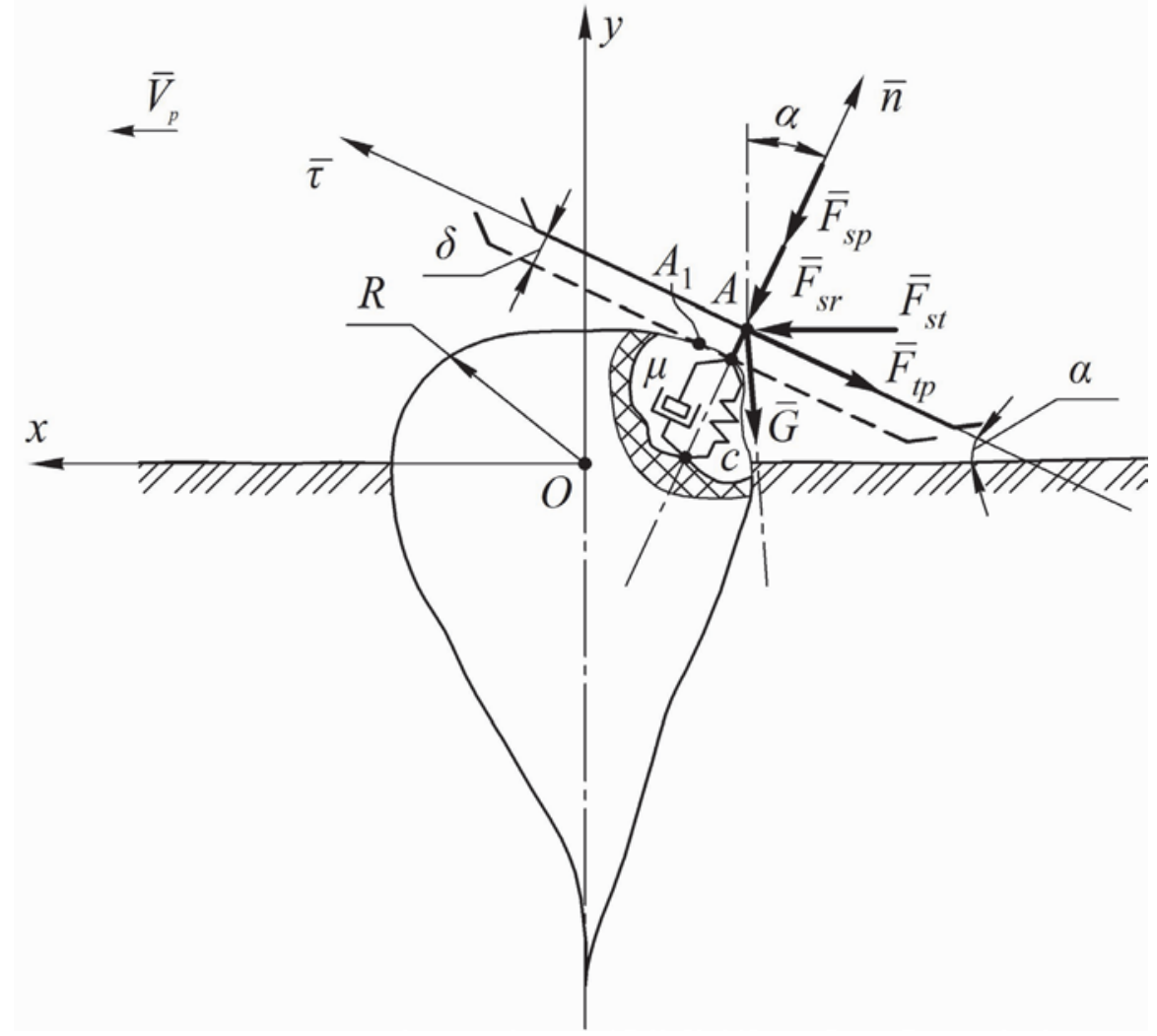

Fig. 1. Equivalent scheme of interaction of passive copier and head of sugar beet root

We pass through a point $A$ a system of rectangular natural coordinates $\epsilon \overline{A n}$, whose axis $\epsilon$ passes through the copier plane at the tangent to the root of the head at the point of contact, and the axis $\bar{n}$ is directed normal to the head of the root crop upwards.

Let us mark all the forces acting on the copier when in contact with the head of the root beet root. This, first of all, is the driving force of the horizontal - $\bar{F}_{s t}$; additional force of the spring $\bar{F}_{s p}$ provided by the design of the trimmer; the force $\bar{F}_{s r}$ taking into account the friction in the joints of the parallelogram mechanism of the trimmer. These three forces are applied at the point $A$ - the point of contact of a passive copier with a beam of remnants of the hinge, $F_{s p}^{-}$and $F_{s r}{ }^{-}$forces attached to the passive flat copy on the normal, that is, located along the axis $n$. To the point $A$ is also the weight $G$ of the copier, directed vertically downward. In addition, at the point $A$ along the normal $n$, the forces of elasticity $F_{c}$ of the hinge and the viscous support ${F_{\mu}}^{-}$are applied. Along the tangential $\epsilon$ applied force $\quad \bar{F}_{t p}$ of friction arising as a result of moving the copier along the root of the root of the beet root

Taking into account the received scheme of forces, on the basis of the fundamental law of the dynamics of the material point, we write the differential equation of motion of the flat copy on the head of the root of the beet in a vector form:

$$
m \bar{a}=\bar{F}_{s t}+\bar{F}_{s r}+\bar{F}_{s p}^{-}+F_{c}^{-}+F_{\mu}^{-}+F_{t p}^{-}+G,
$$

where $\bar{a}-$ accelerating the movement of a flat copy on the root of the root of the beet; $m$ - mass flat copier. 
The motion of the copier will be considered in the absolute immovable system of Cartesian coordinates $x O y$, where the axis $O x$ is directed horizontally to the direction of the copy, and the axis $O y$ is directed vertically upwards, the origin of the coordinates (the point $O$ ) is located in the center of the circle, the upper part of which simulates the shape of the surface of the head of the root

Determine the value of all the forces included in the vector equation (1). Elasticity forces $F_{c}{ }^{-}$and the viscous resistance (damping) ${F_{\mu}}^{-}$will be considered as a result of the deformation $\gamma$ and rate of deformation $\dot{\gamma}$ of elastic residues (petioles) of the hinge and acting in the direction of the normal $n$ to the surface of the root of the root of the beet root.

Moreover, the deformation of elastic remnants of the hinge depends largely on the location of the petioles on the head of the root before contact with the surface of the copier. So, at the beginning of the contact, some petioles may undergo deformation of compression, some bend, and some compression and bend at the same time. However, as soon as the petioles are pressed with a flat surface of the copier to the head of the root, it can be assumed that this bundle of curved petioles will continue to undergo deformation of compression, up to partial jerking. Therefore, it is this bundle of squeezed petioles of the remains of the hinge and will create an elastic-viscous resistance to the direct contact of a flat copy with a spherical surface of the head of the root beet, protecting it from damage. Obviously, in the general case, the magnitude of the deformation $\gamma$ and speed of this deformation $\dot{\gamma} \quad$ may depend on the coordinates $x, y$ the position of the copier, the speed of movement $V$ of the copier on the head of the root and the time $t$ of this movement. Therefore, it is expedient to determine the values of these forces according to the following expressions

$$
\begin{aligned}
& F_{c}=c \cdot \gamma(x, y, V, t), \\
& F_{\mu}=\mu \cdot \dot{\gamma}(x, y, V, t),
\end{aligned}
$$

where $\gamma(x, y, V, t), \quad \dot{\gamma}(x, y, V, t)$ - respectively, the magnitude of the deformation and the rate of deformation of the beam of remnants (petioles) of the hinges, which are simultaneously in contact with the surface of the copier; $c$ - coefficient of elastic deformation of the beam of remnants (petioles) of the hinges, $\mathrm{N} \cdot \mathrm{m}^{-1} ; \mu$-coefficient of viscous resistance (damping) of a beam of remnants (petioles) of a hinge, $\mathrm{N} \cdot \mathrm{s} \cdot \mathrm{m}^{-1}$.

Let's express the magnitude of the deformation $\gamma$ and the rate of deformation $\dot{\gamma}$ of the short remains of the hinge through the coordinates of the position of the copier when it moves along the root of the root of the beet at an arbitrary time.

Let the contact first, when all the pebbles of the bundle of remnants of the hinges that fall into the contact area, pressed the flat surface of the copier to the head of the root, the thickness of the formed layer from the remnants of the hinge will be equal $\delta_{0}$. With further movement of the copier on the root of the root of the specified layer of remnants of the hinge begins to compress. Let at any moment of time the thickness of the layer of compressed remnants of the hinge will be equal $\delta$. Then the deformation $\gamma$ of the indicated layer at this time of time is equal to:

$$
\gamma=\delta-\delta_{0} .
$$

Since the copier moves along the head of the root cordlessly, at an arbitrary time when the copier is at a point $A(x, y)$, the thickness of the layer $\delta$, expressed in terms of the coordinates of the point $A$, will be equal to:

$$
\delta=\sqrt{x^{2}+y^{2}}-R,
$$

where $R$ - radius of head of root beet. 
Given the expression (3), the deformation of the layer of the remainder of the jumper at an arbitrary point $A$ will be determined by the following expression:

$$
\gamma=\sqrt{x^{2}+y^{2}}-R-\delta_{o}
$$

By differentiating the expression (5) by time $t$, we obtain the speed of the specified deformation at any given time. We have:

$$
\dot{\gamma}=\frac{x \dot{x}+y \dot{y}}{\sqrt{x^{2}+y^{2}}} .
$$

Taking into account expression (2), and also expressions (5) and (6), we obtain the strength of the elasticity of the deformation of the compressed beam of the remnants of the hinge in the following form:

$$
F_{c}=c\left(\sqrt{x^{2}+y^{2}}-R-\delta_{o}\right),
$$

and the force of the viscous resistance (damping):

$$
F_{\mu}=\mu(x)\left(\frac{x \cdot+y \dot{x}}{\sqrt{x^{2}+y^{2}}}\right) .
$$

As can be seen from the power circuit (Fig. 1), the frictional force will be equal to:

$$
\left.F_{t p}={ }_{f \mid F_{s p}{ }^{+} F_{s r}-F_{c} \bar{F}_{\mu}}{ }^{-} F_{s t} \cdot \cos (x, n)^{+} G \cdot \cos (y, n)\right] \text {, }
$$

where $f$ - the coefficient of friction of the surface of the residues (petioles) of the hinges on the surface of the plane passive copier.

In projections on the axis $O x$ and $O y$ of coordinate of the vector equation (1) will be written in the form of a system of differential equations of the following form:

$$
\begin{aligned}
& \ddot{m x}=F_{s t}-F_{s r} \cos (x, \hat{n})-F_{s p} \cos (x, \hat{n})+ \\
& +F_{c} \cos (x, \bar{n})+F_{\mu} \cos (x, \bar{n})-F_{t p} \cos (\dot{x}, V),{ }_{\}} \mid \\
& m \ddot{y}=-F_{s r} \cos (y, \vec{n})-F_{s p} \cos (y, n)+F_{c} \cos (y, n)+\mid \\
& +F_{\mu} \cos (y, n)-F_{t p} \cos (\dot{y}, V)-G,
\end{aligned}
$$

where $\cos (\hat{x, \bar{n}}), \cos (y, \hat{n})$ - directional cosines of the vector of normal $n$ to the axes Ox and Oy respectively; - direction cosines of the vector of velocity $V$ to the axes $O x$ and $O y$, respectively; $\cos (\dot{x}, \bar{V}), \cos (\dot{y}, \bar{V})-$ projections of the vector of velocity $\bar{V}$ on the axis $O x$ and Oy accordingly.

Further, according to [13], determining the directional cosines and taking into account that the root head of sugar beet has a spherical shape, we define the function gradient module and the point velocity module:

$$
\begin{gathered}
\otimes f=\sqrt{\left(\frac{\partial f}{\partial x}\right)^{2}\left(+\frac{\partial f}{+}\right)^{2}}, \\
V=\sqrt{x^{2}+\dot{y}^{2}} .
\end{gathered}
$$

Substituting in (10) the values obtained and after certain transformations we obtain the following system of differential equations: 


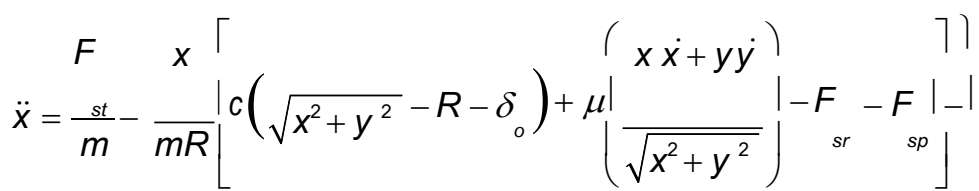

$$
\begin{aligned}
& -\frac{\dot{x f}}{\sqrt{x^{2}+\dot{y}^{2}}}\left[\begin{array}{c}
F+F_{s r}-c\left(\sqrt{x^{2}+y^{2}}-R-\delta\right. \\
x
\end{array}\right)-\mu \mid\left(\frac{x \dot{x}+y \dot{y}}{\sqrt{x^{2}+y^{2}}}\right)- \\
& \left.-\frac{x}{R} F_{s t}+\frac{y}{R} G\right] \text {, }
\end{aligned}
$$

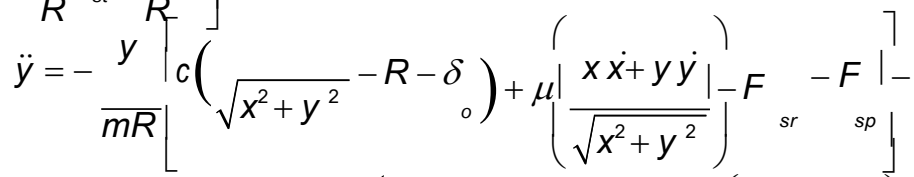

$$
\begin{aligned}
& -\frac{\dot{y f}}{\sqrt{\dot{x}^{2}+y^{2}}}\left[\begin{array}{c}
F+F_{s r}-c\left(\sqrt{x^{2}+y^{2}}-R-\delta\right. \\
s p
\end{array}\right)-\mu\left(\frac{x \dot{x}+y \dot{y}}{\sqrt{x^{2}+y^{2}}}\right)- \\
& -\underline{x}_{F}+\underline{y}_{G}{ }^{\top}-g \text {. } \\
& R^{\text {st }} R
\end{aligned}
$$

This system of differential equations (13) represents the calculated mathematical model of the inaccessible motion of a flat copy in the very part of the head of the root beet, where the most likely was a hard impact, which always leads to damage to the upper part of the root crop of beet or to knocking out all of the root crop from the soil. In addition, the resulting system (13) is a system of differential equations of the second order, which can be solved numerically using a PC and for an individual case, for example, when the forces acting on the operation of this dynamic system have constant and maximum values.

The initial conditions for the solution of the system of differential equations (13) will be the following conditions:

$$
\text { at } t=0: \quad x=x_{0}, \quad y=y_{0}, \quad \dot{x}=V_{p}, \quad \dot{y}=0 .
$$

At the beginning of the contact of the copier with the head of the root crop, the lateral petioles are as deformed as possible, thus creating maximum elastic $F_{\text {cmax }}$ and damping forces $F_{\mu \max }$ that soften the shock load, replacing it with smoother contact interaction.

Taking these comments into account, we shall consider the motion of a copier on the head of the root crop at the very beginning of its contact with the root crop, then for this initial contact area the system of differential equations (13) is greatly simplified and finally we finally get:

a) the law of changing the speed of movement of the copier on the root of the root of the root of the contact:

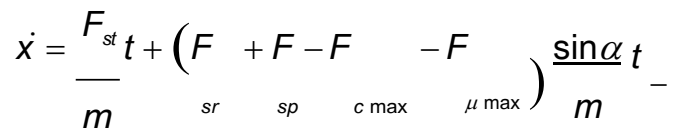

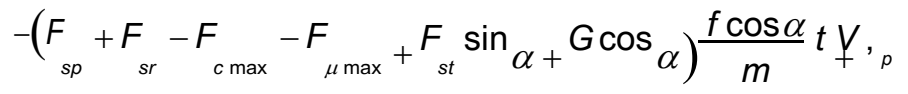

$$
\begin{aligned}
& \dot{y}=\left(F_{c \max }+F_{\mu \max }-F_{s r}^{s r}-F_{s p}\right) \underset{m}{f \sin \alpha} t_{-}\left(F_{s p}+F_{s r}-F_{c \max }-F_{\mu \max }+\mid\right. \\
& \left.+F_{s t} \sin \alpha+G \cos \alpha\right) \frac{f \sin \alpha}{m} t-g t .
\end{aligned}
$$

б) the law of copying the root of the head at the beginning of the contact: 


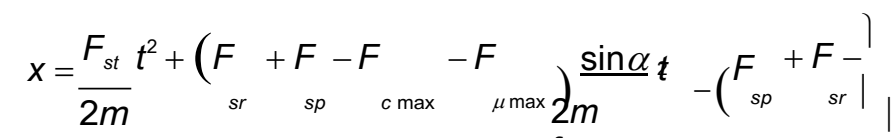

$$
\begin{aligned}
& \left.-F_{c \text { max }}-F_{\mu \max }+F_{s t} \sin \alpha+{ } \cos \alpha\right)^{\frac{f \cos \alpha}{2 m}} t_{2}+{ }_{p} t_{{ }^{+}} x \text {, } \\
& y=\left(F_{c \text { max }}+F_{\mu \text { max }}-F_{s r}-F_{s p}\right) \frac{\cos \alpha}{2 m} t^{2}-\left(F^{s p}+F_{s r}-F_{c \max }-\right. \\
& \left.-F_{\mu_{\max }}+F \sin \alpha+G \cos \alpha\right) \frac{f \sin \alpha}{2 m} t^{2}-\frac{g t^{2^{s p}}}{2}+y_{0}{ }^{c r} \quad{ }^{c \max }
\end{aligned}
$$

In order that the rootstock has not been struck out of the ground during the impact of a flat passive copier on its head, it is necessary to ensure that the root beet does not sow the soil from the ground:

$$
\begin{aligned}
& F_{s t}+\left(F_{s r}+F_{s p}-F_{c \text { max }}-F_{\mu \max }\right) \sin \alpha- \\
& -f\left(F_{s r}+F_{s p}-F_{c \text { max }}-F_{\mu \max }+F_{s t} \sin \alpha+G \cos \alpha\right) \cos \alpha<\left[P_{g}\right] .
\end{aligned}
$$

This inequality serves as a test for any set of forces in its left side. The dependencies of moving a flat copy in the vertical plane along the root of the root of the root at the beginning of the contact are shown in Fig. 2.

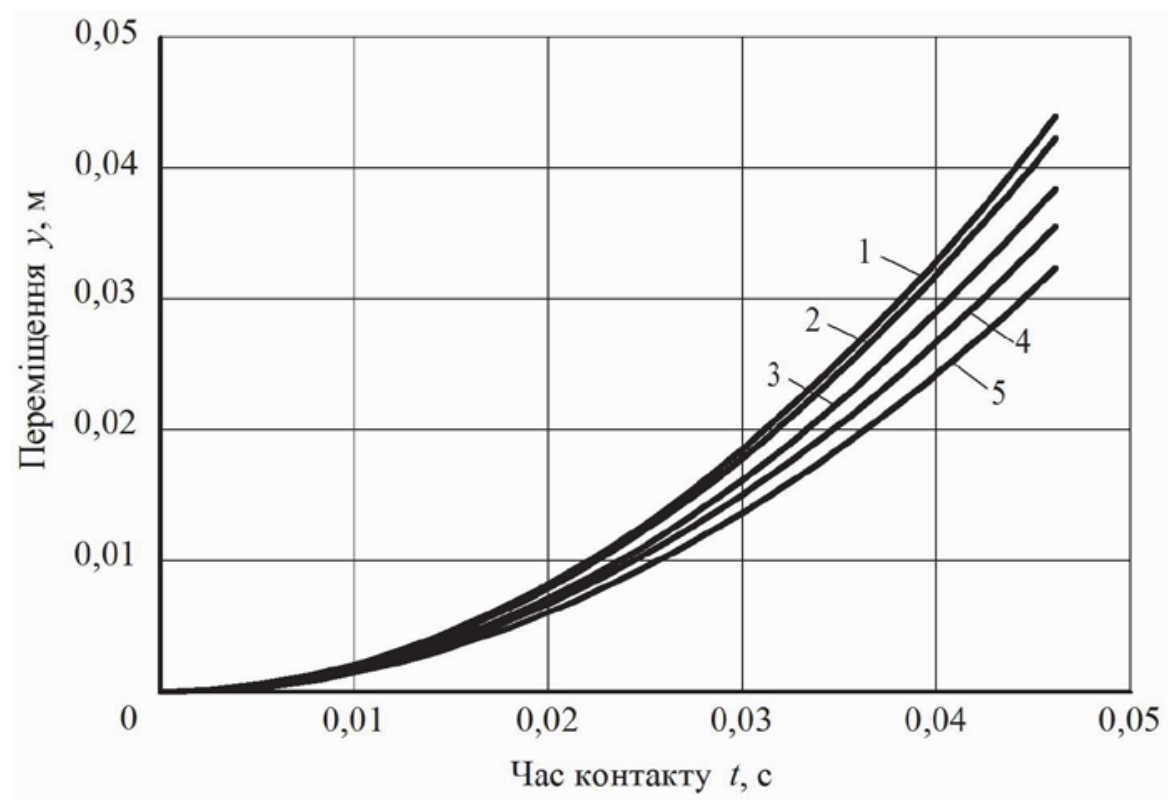

Fig. 2. Depending on the movement $y$ of the copier on the head of the root from the time of contact $t$ at the angle of inclination $\alpha: 1-\alpha=5 \stackrel{o}{;} ; 2-\alpha=15^{\circ} ; 3-\alpha=25^{\circ} ; 4-\alpha=30^{\circ} ; 5-\alpha=35^{\circ}$

Analyzing Fig. 2, it can be concluded that the movement is intensively increasing during the contact of the flat copier with the root sugar of sugar beet, and an increase in the angle $\alpha$ of inclination leads to a decrease in the value of the displacement $y$.

The results of numerical simulation of the obtained system of equations for determining the parameters of a copier during its movement along the head of the root crop at a forward speed of $1 \mathrm{~km}$. h-1 are presented in Fig. 3. 


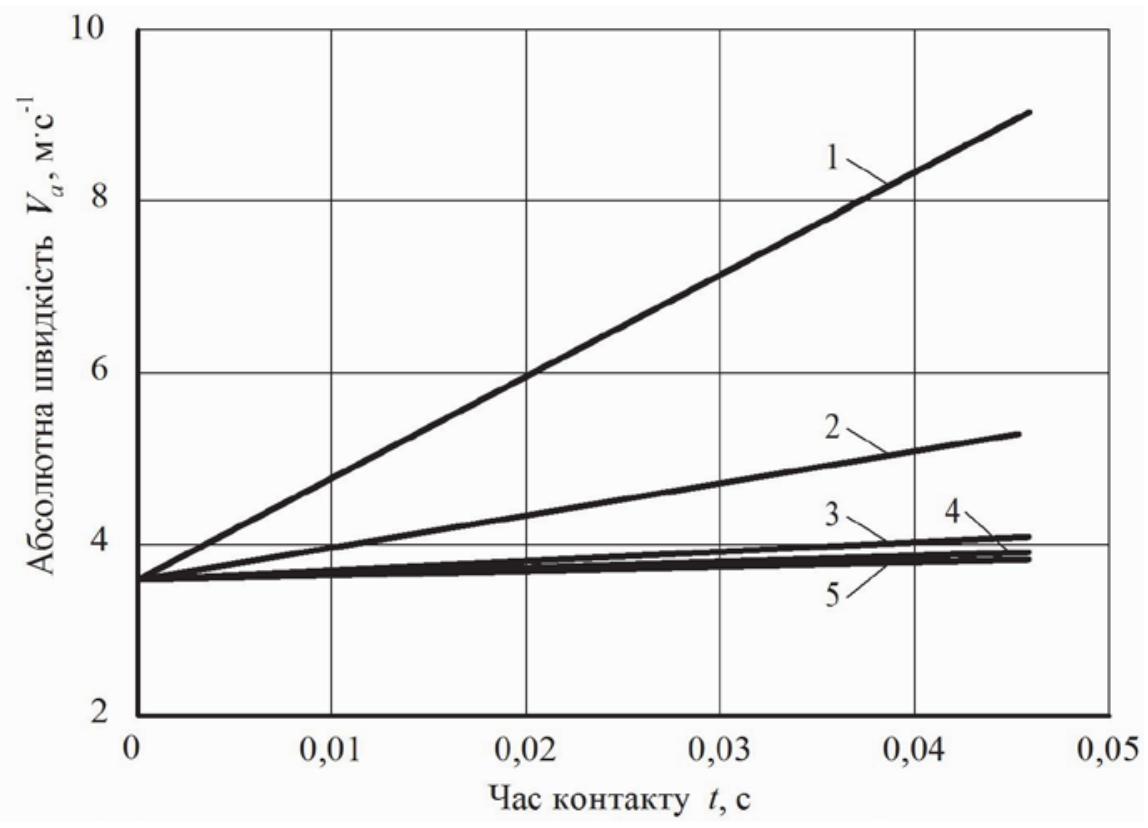

Fig. 3. Dependence of absolute velocity $V_{a}$ of copier from contact time $t$ at angle of inclination $\alpha=5^{\circ}$ for different masses: $1-m=1 \mathrm{~kg} ; 2-m=3 \mathrm{~kg} ; 3-m=10 \mathrm{~kg} ; 4-m=15 \mathrm{~kg} ; 5-m=20 \mathrm{~kg}$

Taking into account that at an initial velocity of $V=3.6 \mathrm{~m} \cdot \mathrm{s}^{-1}$, the value of the absolute velocity $V_{a}$ at the beginning of the contact between the flat copy and the root canal of sugar beet can exceed it by $2 \ldots .2 .5$ times and grow rapidly in a very short period of time, then choose constructive the parameters of the copier and its suspension system, without the above dependencies and the developed mathematical model is not possible. Also, from the analysis of dependencies it is seen that increasing the mass $m$ of a copier to $10 \mathrm{~kg}$ leads to a significant decrease in its absolute velocity, and hence vertical vibrations.

\section{Conclusions}

A new theory of the process of interaction of a passive copier with a root of sugar beet root has been developed. What is the power scheme for the interaction of a passive copier and the spherical surface of the beet root head. In this contact the elastic-dampening properties of the remnants of the hinge (petioles) are taken into account, which are presented in the form of a model with elastic and viscous properties. At the points of contact on the above schemes are applied all the forces in force. Using the basic law of dynamics, a new system of differential equations is presented that describes the motion of a plane passive copier along the spherical surface of the head of the sugar beet root, which contains the remains of the hinge. After double integration, the laws of changing the speed of movement and the movement of a plane passive copy on the root of the root of the root at the beginning of the contact are obtained. In this case, the condition is not to knock out the bodies of root beet from the soil. The use of the new analytical dependencies and the results of specific numerical calculations on the PC in the design and design of beet-harvesting machines will provide significant improvement in the quality of the hips and root crops of sugar beets in their mechanized harvesting.

\section{References}

1. Bulgakov V. Study on the interaction of feeler and roots within the topping process of sugar beet. Bulletin of the Transylvanian University of Braşov. Vol. 9 (44) - New Series. Series A, 2002. - p. 79-84.

2. Pogoreliy L.V. Beet harvesters. Design and calculation / L. V. Pogoreliy, N. V. Tat'yanko, V. V. Bray et al. Ed. L. V. Pogorelogo. - K .: Tekhnika, 1983. - 168 p. 
3. Helemendik M.M., "Directly and Methodically, the Separation of Novel Robotic Organisms of Sils'kospodarskih Machines." MM Khelemendik. - K .: Agrarna Nauka, 2001. - 280 p.

4. Bendera I.M. Substantiation of the method and design of the machine for separating the hinge from the root crops of sugar beet / I. M. Bender, M. M. Boris, A. M. Boris // Vysn. Lviv. NAU: agroengineering research. - Lviv: NAU, 2008. - No. 12 (2). - 762 p.

5. Lynnyk A. Determination of dynamic parameters of a rigid cleaner in interaction with roeplopodom / A. Linnik // Bulletin TNTU. - 2014. - Volume 73. - No. 1. - P.165-171

6. Boris A.M. Modeling of the technological process of removing the hook by a combined method / AM Boris // News of agrarian science. - 2011. - №7. - P. 66-68.

7. Smith $L$. The effect of defoliator flail configuration, speed and crown removal on sugarbeet yield, quality and profitability / L. Smith // Sugarbeet Research and Extension Reports. - 1991. - Vol. 22. P. 222-227.

8. V.Ya. Martynenko, "Justification of the constructive parameters of head cleansers for root crops," V. Ya. Martynenko, "Proceedings of the International Scientific and Practical Conference: Problems and Perspectives of the Creation of Beet Harvesting Equipment." - Vinnitsa, 1996. - P. 41-44.

9. Gurchenko A.P. Mechanization of harvesting of sugar beet tops / AP Gurchenko, Ya.V. Savchenko // Engineering in agriculture. - Moscow: The harvest, 1986. - No. 9. - P. 15-17.

10. Ihnatiev Ye.l. Development of new constructive and technology scheme of sugar beet tops harvesting with use of arable and row-crop tractor / Ye.

11. Bosoy E.S. Theory, construction and calculation of agricultural machines: a textbook for higher educational institutions of agricultural purpose / E.S. Bosoy, O.V. Vernyaev, I.I. Smirnov, E.G. Sultan-Shah; Ed. E.S. Bosoy. - 2 nd ed. Pererab. and additional. - Moscow: Mechanical Engineering, 1978. - 568 p.

12. Butenin N.V. Course of theoretical mechanics / N.V. Butenin, Ya.L. Lunts, D.R. Merkin. - Volume 2. - Moscow: Science, 1985. - $496 \mathrm{p}$.

13. Vasilenko P.M. Introduction to agricultural mechanics / P.M. Vasilenko. - Kiev: Agricultural Education, 1996. - $252 \mathrm{p}$.

14. Pogorely L.V. Beet harvesters: history, construction, theory, forecast / L.V. Pogorelyi, N.V. Tat'yanko. - K .: Phoenix, 2004. - 232 p.

15. Bulgakov V.M. Beet harvesting machines. Monography / V.M. Bulgakov. - Kiev: Agrarian Science, 2011. - $351 \mathrm{p}$. 